\title{
Detecting Intrusions in Smart Card Applications using Expert Systems and Neural Networks
}

\author{
Thomas Alexandre and Patrick Trane \\ Recherche et Développement Dossier Portable (RD2P-LIFL) \\ 1 , rue du Professeur Jules Leclercq \\ 59037 LILLE Cedex \\ FRANCE
}

In addition to the traditional passwords or Personal Identification Number (PIN) required for getting accesses to the ressources of Smart Cards, we have investigated several ways to complete this basic security scheme by providing tools for detecting intrusions. Because sometimes due to negligence or violence the PIN is not sufficient to guaranty the card's security, our interests have focused on systems specialized in detecting abnormal access and use of the card.

In prior studies we have proposed two implementations of what we called "The Radar Concept" [ALEX94a] [ALEX94b], one using an Expert System for analyzing the data coming from credit card transactions and the other using a Neural Network for achieving the same task.

The goal of such systems was to identify the behavior of someone doing transactions with his card and eventually refuse the required service because that behavior was considered abnormal. In this paper, we present a new form of intrusion detection. Instead of identifying the card user's behavior, we have experimented the recognition of some common intrusion scenarios to limit the card's fraudulent use.

In a first part we analyze the advantages and drawbacks of the Radar intended to recognize users behavior. In a second part, we introduce an implementation of intrusion scenarios detection. We then compare both approaches in terms of efficiency, security and performances. 


\section{The main idea behind "Radar"}

In houses or buildings, radar-based alarms are often used for detecting intrusions. The role of a radar is not to prevent intrusions but to detect abnormal ones. Such radars do not replace keys or secret codes. They just complete and reinforced the security scheme. We have intended to apply this concept in the smart card environment, associating keys to passwords (or PIN) and radars to artificial intelligent systems for controlling the processes attached to applications. A password is independent of the application whereas a Radar is closely related to the application.

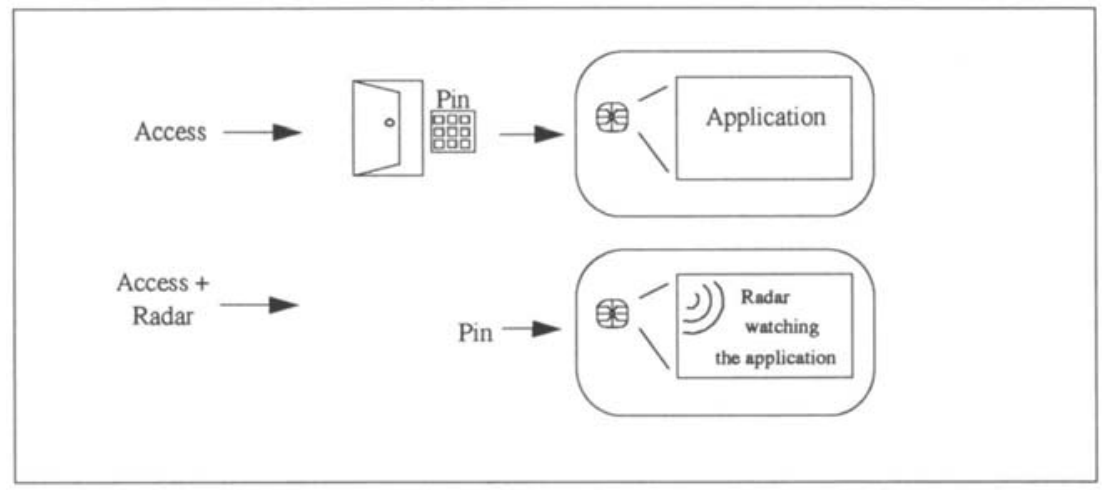

FIGURE 1 The Radar positioning in the Smart Card Security Scheme

The application example we have retained for our experiments was based on credit card transactions and intended to detect abnormal credit card uses.

\section{Modelizing somebody's behavior}

In the prior studies of how to identify a card user's behavior related to a bank credit card application, we have implemented 4 different criteria:

$<$ Nature, Amount, Date, Location>

- Nature: 8 possible natures of a Transaction (Cash, Transportation, Gas, Food, Housing, Furniture, Hobby, Other).

- Amount: a positive number corresponding to the transaction's amount.

- Date: date of the transaction in the form of Month/Day/Year.

- Location: location of the transaction.

Obviously people all have different habits and trying to summarize them considering these 4 elements could only lead to a general system trained to detect irregularities on restricted data. Although these systems have proven their relative efficiency on a 
small set of experiments related to both existing credit card accounts and randomely generated transactions [ALEX94a], they suffer from a number of features which we are going to adress:

\section{For the Expert System:}

- The restricted number of rules that can be implemented in a smart card results in a limited expertise. Increasing the number of items for the checking mechanism tends to rapidly extend the number of rules. For instance, with 4 items <Nature, Amount, Date, Location>, we had constructed the 15 possible rules associated with these single parameters [ALEX94a]:

- 4 rules involving only one item (for example: if Amount $>$ Max_Amount then Alarm++ ).

- 6 rules involving two items at a time:

For example: if Nature $=$ Cash and Amount $>$ Max_Amount then Alarm ++ .

-4 rules involving three items to a single transaction.

- 1 rule involving all of the four items.

Having 5 items instead of 4 would produce a total of 31 rules. The number $\mathbf{N}$ of possible rules applied on $\mathbf{n}$ items is actually given by:

$$
N=\sum_{p=1}^{n} \frac{n !}{p ! \times(n-p) !}
$$

Because of the limited smart card capacity in terms of memory space and processing power, an expert system kernel inside such a card could only handle a few rules to respond within a reasonable time.

- Considering a long term period (here we mean in the order of 6 months) the user's behavior might change whereas the expert system rules remain the same, unless you update the user's knowledge base which means a new enrollment phase in a secure environment similar to the card issue. The difference between the static embedded knowledge base and the dynamic user's behavior evolution can lead to poor reliability after a certain amount of time.

- Once the expert system model has been set up and implemented, any change in the transaction items involves redesigning at least part of the expert system. This could appear if the required information for recording a transaction were changing. This case has been encountered by American Express in a similar problem, when they built an Expert System to automate up to 90 percent of their card verification requests [GOLD90] [ESSI90]. Once 
they had their "Authorizer" [PIKE87] [DZIE89], it became much harder to change their corporate goals. An example of corporate goal changing, is the desire to expand the usage of the American Express card by reducing the requirements to get a card. This goal might increase the acceptable rate of allowing a stolen card to be used, or it might change some other statistical fact used by the expert system.

For the Neural Network (see [GELE91][GARD93] for a general introduction to Neural Networks):

The first thing is the evolution of the behavior pattern. One of the major characteristics of Artificial Neural Networks is a learning phase which consists of teaching the network how to process a particular task. This makes possible later for the network to take into account changes in the behavior pattern. This training algorithm can be performed each time a new transaction is achieved by a card user but at least needs to take place once at the begining on a set of typical transactions performed by a user.

Despite the fact that this interesting particularity of Neural Networks might overcome the problem of behavior evolution encountered with the Expert System solution, other problems seem to appear:

- the training process is time consumer and therefore implementing it in Smart Cards remains difficult. Having this procedure outside the card at the card issue loses the ability for the user's behavior pattern to evolve after each transaction.

- assuming the training process to fit into the smart card also has its drawbacks, mainly because of the complexity of such a training. The most popular algorithm for training a neural network, the Back-propagation [RUME86], by itself is a stable algorithm. However, unless you hit upon the exact right feature set, it is hard to guarantee that your network will converge in a way that will generalize to new problems. This becomes worse if you diverge from standard back-propagation. This problem has been investigated by NeuralWare when setting up Chase Manhattan's credit-card fraud Neural Network [KEYE92]. They looked at unsymetric error functions to bias the network increasingly towards one type of error (allowing a card to be fraudulently used) over other errors (not allowing a customer to use its card).

Because of these few lacks in the user's behavior recognition, we have invetigated other approaches of Radars. One of them involves the recognition of some commonadmitted intrusion scenarios instead of the recognition of a user's behavior. As we obviously can't accurately modelize an intruder's behavior, this last solution may be an alternative to limit the intruder's access. Relevant work on this subject has also been completed in the NIDES project (Next generation Intrusion Detection Expert System) and can be found in [ANDE93] and [JAGA93]. 


\section{Intrusion scenarios recognition}

Our experiments related to intrusion scenarios have been established on the basis of a survey involving different people.

The purpose of this poll was to ask some people how they would use a stolen card if they also had the corresponding PIN. By listing their 15 first transactions, we were able to classify and identify some common scenarios of fraudulent use.

Each of the 15 transactions were described with the 4 parameters already associated with previous Radar studies and mentioned in the preceding section, i.e. $<$ Nature, Amount, Date, Location>. below:

Typical frequently appearing scenarios attached to different attacks are presented

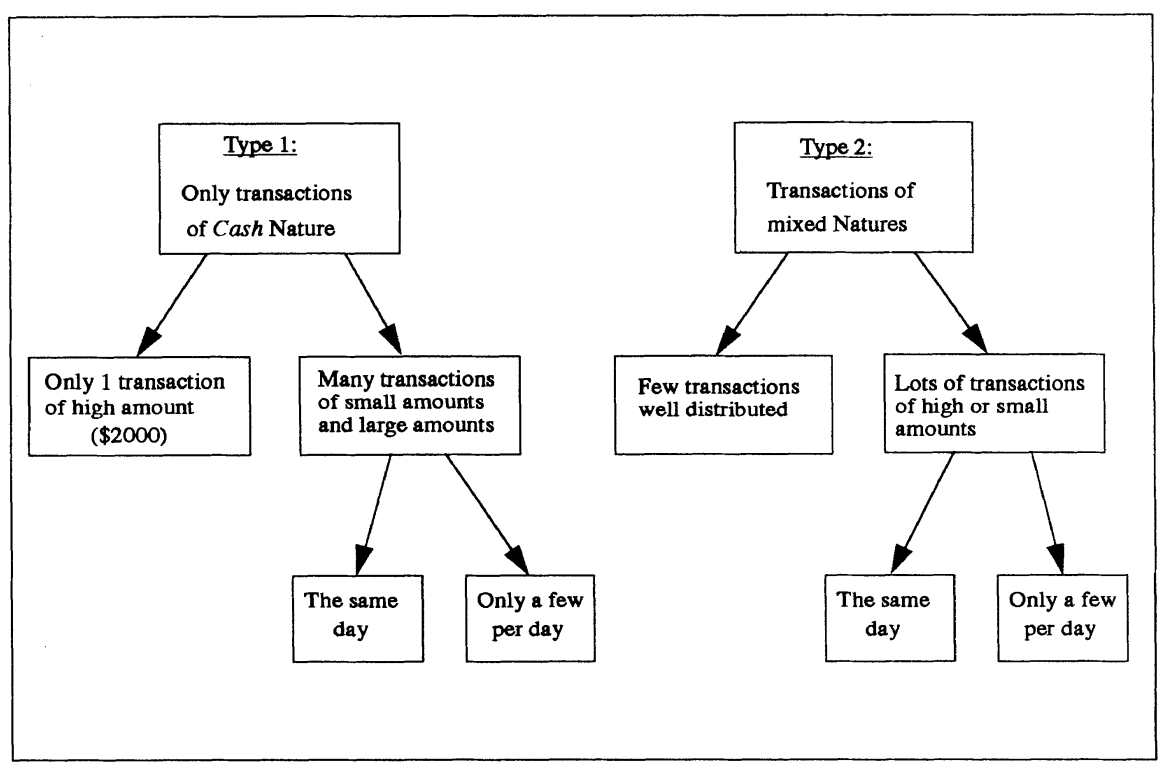

\section{FIGURE 2 A few common intrusion scenarios}

These results have suggested a few immediate comments:

- The major part of fraud concerns transactions of Cash Nature (i.e. getting Cash from dispensers).

- The intruder's behavior is often characterized by the large amount of transactions he is trying to perform within a short time. This is not sufficient to establish that somebody is doing fraudulent use of the card (for example before Christmas a user can suddenly buy a lot of things with his credit card). 
- It is hard to protect the first scenario which is to perform only one high-amount transaction and then throw the card away, because a normal user should be able to perform at least one high-amount transaction without having his card muted. But in that case, the overall fraud is limited.

- The people who might know the owner of the card they have stolen are probably those who are the most difficult to detect, as they might imitate the owner's habits. Their fraud is however limited by the fact that they can't get a lot more money than what the card's owner uses to spend during a short time.

\section{The Intrusion Scenarios Detector}

In this part we present an approach of Radar associated with the problem of detecting some common intrusion scenarios. The goal of such a system is not detect all the frauds associated with abnormal uses of Smart Cards and never will, but is at least designed to reduce the most often encountered intrusions, especially those involving large amounts of transactions achieved within a short time.

We have implemented both a solution related to Expert Systems and a solution based upon Neural Networks.

\section{1. Solution with the Expert System}

Considering the remarks formulated in the previous part about typical intrusion scenarios, we have constructed a few rules using the Prolog language [BRAT90] [SPEN91] in the form of :

$$
\begin{aligned}
& \text { if (predicate) then accepted transaction. } \\
& \text { suspicious transaction. } \\
& \text { refused transaction. }
\end{aligned}
$$

For examples:

- if (Number_of_Cash_Withdraws_the_same_day > 10) then suspicious_transaction.

- if(Amount $>3000$ and Amount_already_spent_the_same_day $>4000$ ) then suspicious_transaction.

We can easily construct rules combining several aspects simultaneously involving information collected during preceding transactions so that the system can take into account parameters related to time intervals. The complexity of the expert system can be adjusted depending of the available size we have on the smart card. This complexity is directly linked with the number of rules added to improve the detection accuracy.

The card thus contains a knowledge base with a few information that are the thres- 
holds upon which alarms are generated. An example of such Knowledge Base is given below:

Table 1: Knowledge-base associated to the Expert System

\begin{tabular}{|c|c|c|c|c|}
\hline Nature & $\begin{array}{c}\text { Number of } \\
\text { Transactions } \\
\text { allowed the } \\
\text { same day }\end{array}$ & $\begin{array}{c}\text { Amount } \\
\text { allowed per } \\
\text { Transaction }\end{array}$ & $\begin{array}{c}\text { Amount } \\
\text { allowed the } \\
\text { same day }\end{array}$ & $\begin{array}{c}\text { Amount } \\
\text { allowed the } \\
\text { same week }\end{array}$ \\
\hline Cash & $<3$ & $<\mathbf{1 5 0 0}$ & $<\mathbf{3 5 0 0}$ & $<\mathbf{6 0 0 0}$ \\
\hline $\begin{array}{c}\text { Other than } \\
\text { Cash }\end{array}$ & $<\mathbf{1 0}$ & $\mathbf{< 1 0 0 0 0}$ & $<\mathbf{1 1 0 0 0}$ & $<\mathbf{1 5 0 0 0}$ \\
\hline
\end{tabular}

The Expert System comprises only a few rules and therefore can fit into a Smart Card, even if the card-embedded expert system kernel built for this purpose and based on the Prolog language still requires quite a powerful card, such as the one described in [PEYR94].

We have tested this system on transactions coming from existing bank accounts and transactions coming from the survey previously evocated, leading to an approximate $80 \%$ of correct fraud detection after 3 transactions. Less than $5 \%$ of genuine users were flagged as fraudulent. This last figure is still very big compared to acceptable rates in the case of bank applications. That's why in this case the system should not mute the card if an alarm is generated but rather resort to the concept of Challenge introduced later in this paper.

\section{4 . 2. A Neural Network based approach}

The Neural Network approach has been set up using the Perceptron algorithm [ROSE57] [MINS69] and Back-propagation algorithm [RUME86] [VOGL88] and presents interesting results in terms of code compactness and processing time.

The training phase doesn't need to take place inside the card because the intrusion scenarios' patterns are not evolving each time a transaction is achieved as for the user's behavior recognition. Therefore the training phase is only performed at the card issue.

We have trained and tested different neural network architectures with different learning rules. The following description of network is one example among them that uses a popular learning rule (back-propagation) and remains a simple implementation with only 6 inputs i.e. 6 criteria for detecting fraud.

The network, consisting of 6 inputs fed to a two-layer network, is trained with backpropagation on a 10 pattern problem. Here we have only used 10 patterns for training to simplify the problem but we could put a larger set of training examples to make the whole system more reliable

Layer 1 consists of 5 tan-sigmoid neurons, while layer 2 is made of one linear neuron. The network is initialized with the Nguyen-Widrow method [NGUY90]. An adap- 
tive learning rate which adjusts to be as large as possible without taking too large of steps speeds up the network while avoiding large increases in error. The momentum technique to avoid being trapped in local minimas is also implemented.



\section{FIGURE 3 A Radar based on Neural Networks}

We have written all the network description and the training algorithm using the Matlab programming language [DEMU92].

The simulation results are summarized in the following diagrams:

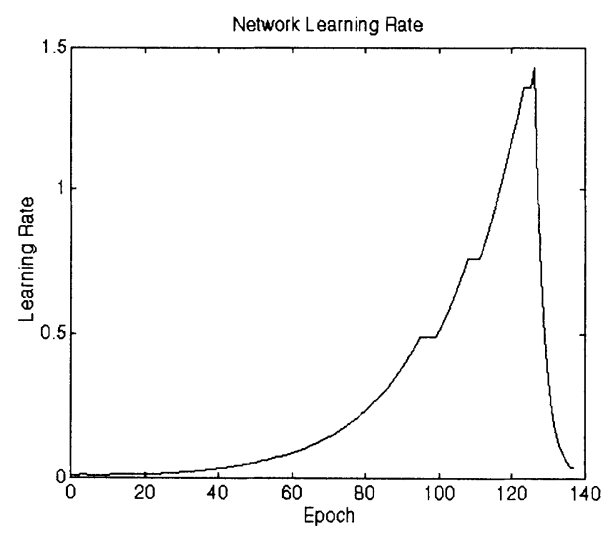

FIGURE 4 Learning rate evolution during the training process 




FIGURE 5 Training phase results

Training took 137 epochs to correctly classify the data, representing about 10 seconds on a 486 microprocessor running $50 \mathrm{Mhz}$.

We have tested this newly trained network on new figures both coming from new transactions achieved by the card owner and transactions completed by intruders, leading to an approximate $90 \%$ of success. This evaluation has only concerned a few transactions to give an idea of the usefulness of the detection process but should be further experimented on additional intrusion strategies to get a more precise performance estimation. 
The memory space required for the network storage consists of $6 \times 5$ weight values +5 biases for the first layer and 5 weights +1 bias for the second layer. Assuming weights and biases are stored as real values ( 4 bytes), the total network size to be recorded on the EEPROM of a smartcard represents 164 bytes.

To evaluate the performance of the feed forward process completed on a smartcard, we have examined an existing smartcard algorithm written for the CASCADE project, which is the definition of a smartcard 32-bit RISC processor featuring biometric recognition based on neural networks [PEYR 94]. We have estimated that the Radar algorithm (feed forward process) applied on such a processor would only consume less than 300 bytes of ROM and less than 30 bytes of RAM, one feed forward process time being about 5000 clock cycles.

\section{3 . Comparison of both approaches}

The neural network implementation has got a few essential advantages over the expert system solution:

- The memory space required for storing the neural network weights and the feed forward processing is a lot smaller than for storing rules and achieving expertise.

- The processing needed for detecting intrusion is also much faster for the neural network alternative.

- An interesting characteristic is the ability for a neural net to generalize to new problems never encountered during the training process.

Although the neural network solution experimented here seems very promising for detecting frauds at a minimum cost in terms of processing power and memory size, there are still a few optimizations that can added. For example, the network size and architecture can be optimized depending on the examples set so that generalization will give better results of detection.

\section{4. The «Challenge $*$ concept}

A 'Challenge' proposed by the card might solve the problem of uncertainty associated with the card decision on some particular cases, i.e. when the fraud detector still has a doubt on the card user's identity after processing its radar. In such cases, the challenge the card would give to the user as an additional authentication could consist of a question related to the card owner's private life. This could be for example a question like:

- What is your favorite rock singer?

- Where did you spend your most memorable vacation?

Such a question can be reminded by the owner without any effort (unlike passwords) and could be chosen randomely in a set of questions recorded into the card at the 
card issue, whereas it seems unpractical for the intruder to know such precise answers.

\section{Conclusion}

In order to effectively address the various intrusion threats, a system should combine several intrusion-detection approaches, as emphasized in the NIDES project [LUNT93].

We are now investigating additionnal implementations of fraud detectors based on Neural Networks and especially using unsupervised learning techniques similar to Kohonen's Self-Organizing Maps [KOHO87]. These methods help on finding the relevant criteria to give as inputs of supervised neural nets such as those described in the preceding part.

\section{REFERENCES}

[ALEX94a] Thomas Alexandre, Vincent Cordonnier, "The Radar Concept", in Proceedings of the Cardtech/Securtech'94 International Conference, Arlington, Virginia, USA, April 10-13, 1994, p87-98.

[ALEX94b] Thomas Alexandre, "The Radar Concept using Neural Networks", in Proceedings of the CARDIS'94 First Smart Card Research and Advanced Application Conference, Lille, France, October 24-26, 1994.

[ANDE93] D. Anderson, T. F. Lunt, H. S. Javitz, A. Tamaru, A. Valdes, "Detecting Unusual Program Behavior Using the NIDES Statistical Component", Safeguard Final Report, SRI International, Menlo Park, California, December 1993.

[BRAT90] Ivan Bratko, Prolog Programming for Artificial Intelligence, second edition, Addison-Wesley, 1990.

[DEMU92] H. Demuth, M. Beale, Neural Network Toolbox for Use with Matlab, The MathWorks Inc., 1992.

[DZIE89] J. M. Dzierzanowski, K. R. Chrisman, G. J. Mac Kinnon, P.Klahr, "The Authorizer's Assistant: a knowledge-based credit authorization system for American Express", in Proceedings of the First Annual Conference on Innovative Applications of Artificial Intelligence, Stanford, CA, USA, March 28-30, 1989.

[ESSI90] J. Essinger, "Artificial Intelligence in retail banking: two key case studies", Financial Technology International 1991 Guide, IBC Business 
Publishing, London, UK, 1990.

[GARD93] Daniel Gardner, Neurobiology of Neural Networks, The MIT Press, 1993.

[GELE91] E. Gelenbe, Neural Networks: Advances and Applications, Elsevier Science Publishers, 1991.

[GOLD90] N. M. Goldsmith, "Beyond Technology: management expert system projects at American Express Company", in Proceedings of the IEEE Conference on Managing Expert Systems Programs and Projects, Bethesda, MD, USA, Sept. 10-12, 1990.

[JAGA93] R. Jagannathan, A. Tamaru, F. Gildham, D. Anderson, C. Jalali, C. Dodd, H. S. Javitz, A. Valdes, T. F. Lunt and P. G. Neumann, "Next Generation Intrusion-Detection Expert System (NIDES): Software Design Specifications", Technical Report, Computer Science Laboratory, SRI International, Menlo Park, California, March 1993.

[KEYE92] J. Keyes, "Winning back investors' confidence", Information Strategy, Vol.8, no.2, Winter 1992.

[KOHO87] Teuvo Kohonen, Self-Organization and Associative Memory, 2nd edition, Berlin: Springer-Verlag, 1987.

[LUNT93] Teresa F. Lunt, "Detecting Intruders in Computer Systems", in Proceedings of the 1993 Conference on Auditing and Computer Technology, 1993.

[MINS69]Ｍ. Minsky, S. Papert, “Perceptrons”, The MIT Press, 1969.

[NGUY90] D. Nguyen, B. Widrow, «Improving the learning speed of 2-layer neural networks by choosing initial values of the adaptive weights», International Joint Conference on Neural Networks, Vol.3, pp 21-26, July 1990.

[PEYR94] P. Peyret, "RISC-based, Next-generation Smart Card Microcontroller Chips", in Proceedings of the CardTech/SecurTech' 94 International Conference, Arlington, Virginia, USA, April 10-13, 1994.

[PIKE87] L. Piketty, J. Bakin, F. K. Dashiell, J. M. Dzierzanowski, "The Authorizer's Assistant: a large commercial expert system application", in Proceedings of the Third Annual Artificial Intelligence and Advanced Computer Technology Conference, Long Beach, CA, USA, April 22-24, 1987.

[ROSE57] F. Rosenblatt, "The Perceptron: a Perceiving and Recognizing Automaton", Project PARA, Cornell Aeronautical Lab. Report 85-460-1, 1957.

[RUME86] D. E. Rumelhart, G. E. Hinton, R. J. Williams, "Learning internal representation by error propagation", D.Rumelhart and J.Mc Clelland editors, Parallel Distributed Processing: exploring the Microstructure of Cognition, Vol.1, M.I.T. Press, Cambridge, MA, 1986. 
[SPEN91] R. Spencer-Smith, Logic and Prolog, AI Group, Middlesex Polytechnic, A Harvester Wheatsheaf Publication, 1991.

[VOGL88] T. P. Vogl, J. K. Mangis, A. K. Rigler, W. T. Zink, D. L. Alkon, “Accelerating the convergence of the bakpropagation method", Biological Cybernetics, Vol. 59, pp 257-263, 1988. 